\title{
Fisetin attenuates Renal Ischemia/Reperfusion Injury by improving mitochondrial quality, reducing apoptosis and oxidative stress
}

\section{Priyanka N Prem}

SASTRA Deemed University: Shanmugha Arts Science Technology and Research Academy

Gino A Kurian ( $\sim$ kurian@scbt.sastra.edu )

SASTRA Deemed University: Shanmugha Arts Science Technology and Research Academy

\section{Research Article}

Keywords: Fisetin, renal ischemia reperfusion injury, mitochondria, mitochondrial bioenergetics, mitochondrial dynamics

Posted Date: October 26th, 2021

DOI: https://doi.org/10.21203/rs.3.rs-937139/v1

License: (c) (i) This work is licensed under a Creative Commons Attribution 4.0 International License.

Read Full License

Version of Record: A version of this preprint was published at Naunyn-Schmiedeberg's Archives of Pharmacology on February 8th, 2022. See the published version at https://doi.org/10.1007/s00210-02202204-8. 


\section{Abstract \\ Purpose}

Renal ischemic reperfusion (IR) injury is one of the major source of mortality and morbidity associated with acute kidney injury (AKI). Several flavonoids have shown to be renal protective against many nephrotoxic agents causing AKI. Fisetin, a promising flavonoid, is effective in the management of septic $A K I$, expected to ameliorate renal IR injury. Present study aim to generate evidence for fisetin mediated renal protection against IR injury

\section{Methodology:}

Male wistar rats of 200-250 g subjected to IR protocol by performing bilateral clamping for 45 minutes and reperfusion for 24 hours. Fisetin administrated 30 minutes ( $20 \mathrm{mg} / \mathrm{kg} \mathrm{b.wt,} \mathrm{ip)} \mathrm{before} \mathrm{the} \mathrm{surgery.}$ Renal injury was evaluated by measuring the biomarkers in plasma, examining the ultrastructure of kidney and analyzing the apoptotic changes. Oxidative stress, antioxidant levels and mitochondrial function were analysed in the renal tissue.

\section{Results}

Fisetin administration significantly reduced the renal damages associated with IR, by improving estimated glomerular filtration rate (eGFR: IR-0.35 ml/min, F_IR-9.03 ml/min), reducing plasma creatinine level (IR-2.2 mg/dl, F_IR-0.92 mg/dl) and lowering urinary albumin/creatinine ratio (IR-6.09 F_IR-2.16), caspase activity, decreased DNA fragmentation and reduced tubular injury score (IR-11 F_IR-6.5). At cellular level, fisetin significantly reduced renal oxidative stress and augmented the antioxidant levels. Fisetin found to preserve mitochondrial electron transport chain activities and improved the ATP producing capacity in the renal tissue upon IR injury.

\section{Conclusion}

fisetin pre-treatment attenuate renal IR injury by improving the renal function, reducing the renal injury mediated by apoptosis, reducing free radical release, and augmenting mitochondrial function.

\section{Introduction}

Renal insufficiency due to ischemia reperfusion (IR) is one of the major concerns of clinicians in the management of acute kidney diseases where IR injury can compromise normal regulation of fluid, electrolyte and acid base homeostasis (Basile et al. 2012; Ding et al. 2017). This inevitable injury may occur after infarction, sepsis, organ transplantation that leads to acute kidney injury by exacerbating tissue damage by triggering inflammatory cascade, reactive oxygen species and inactivation of 
mitochondrial function (Ding et al. 2017). Long-term and short-term complications of AKI are associated with IR (Doyle and Forni 2016). The search for a suitable therapeutic agent to attenuate the clinically challenged IR is still continuing.

Evidence from the literature testifies the beneficial effects of different agents in combating IR injury. The major groups of molecules includes anti-apoptosis/necrosis agents, free radical scavengers, antisepsis, growth factors, vasodilators and anti-inflammatory agents (Jo et al. 2007). Although these compounds showed beneficial effects in pre-clinical settings, clinical translation of its effect is questionable as the compounds look for specific targets and it is difficult to rule out other overlapping or similar effects that could have been partly responsible for causing renal injury and dysfunction (Jo et al. 2007). This may be addressed by increasing the ability of the renal tissue to withstand IR or search for a multi targeted agent to tackle multifaceted IR pathology.

Natural products like flavanoids are drawing increasing attention for the treatment of renal diseases, primarily due to its ability to trigger multiple targets apart from its antioxidant potential (Vargas et al. 2018). Flavonoids are reported to have antihypertensive, antidiabetic, anti-carcinogenic and antiinflammatory effects (Panche et al. 2016). Many of them exert reno-protective actions in diseases such as glomerulonephritis, diabetic nephropathy, and chemically-induced kidney insufficiency (Arab et al. 2016; Vargas et al. 2018). Flavonoids are known to prevent renal injury associated with arterial hypertension, both by decreasing blood pressure and by acting directly on the renal parenchymal cells (Bai et al. 2013; Vargas et al. 2018). These outcomes are resulting from their interaction with multiple signaling pathways known to produce renal injury.

Fisetin (3, 7, 3,4-tetrahydroxyflavone) is a flavonoid widely present in many fruits and vegetables with the highest concentrations in strawberries (Khan et al. 2013). Fisetin is already reported to have several pharmacological benefits including anti-inflammatory, anti-apoptotic, antioxidant, anti-tumorigenic and anti-angiogenic effects (Antika and Dewi 2021). Physicochemical analysis of fisetin indicated that the compound as well as its metabolites had a high concentration in mouse kidneys compared to other organs, suggesting its potential in treating renal diseases (Touil et al. 2011). The reno-protective effects of fisetin have been confirmed in animal models of diabetic nephropathy, cisplatin-induced nephrotoxicity and LPS induced septic AKI (Prasath and Subramanian 2011). Fisetin's ability to ameliorate IR injury has been proven in organs such as heart, liver and brain (Shanmugam et al. 2018; Li et al. 2021; Zhang and Cui 2021). Fisetin has been reported to exert IR protective activity in the heart via the PI3K/Akt/GSK3 $\beta$ pathway (Shanmugam et al. 2018) and by reducing oxidative stress and inflammation in liver and brain (Li et al. 2021; Zhang and Cui 2021). IR is a bioenergetic related disorder rooted in parenchymal or stromal cells, where these cells varied in different organ and thus have different impact and involve distinct mediators (Kisseleva and Brenner 2008). However, limited information is available about its IR protective role in renal ischemia reperfusion.

The present study aims at unravelling the reno-protective effects of fisetin and underlying mechanisms in a rat model of renal ischemia reperfusion injury. 


\section{Material Methods}

\section{Animals}

Male wistar rats (230-250 g) were obtained from Central Animal facility, SASTRA deemed to be University. Animals were kept for at least one week prior to the experiments in the animal unit under standardized conditions of temperature $\left(22 \pm 1^{\circ} \mathrm{C}\right)$, humidity $(55 \pm 5 \%)$ and $12 \mathrm{~h} / 12 \mathrm{~h}$ light/dark cycles with free access to food and water.

\section{Anesthesia, analgesia and surgical procedure}

All the animal experiments carried out are in compliance with the guidelines of Committee for the Purpose of Control and Supervision of Experiments on Animals (CPCSEA) (552/SASTRA/IAEC/RPP). Anesthesia with isoflurane and analgesia with meloxicum were used for the surgical procedure. The vascular pedicle of each kidney was mobilized. In animals undergoing clamping both renal pedicles were occluded using atraumatic mini-bulldogs. Subsequent to the ischemic period, the reperfusion attained by removing the clamps and perfusing the kidney. Animals were sacrificed by cardiac incision under deep isoflurane anesthesia at the end of the experiment.

In this study, rats were randomized into 6 groups consisting of 6 rats in each group. I) Sham(S): all procedures remained similar except the occlusion of renal arteries was not carried out. II) Fisetin Sham(F_S): Surgical procedures were similar to sham. Fisetin $(20 \mathrm{mg} / \mathrm{kg})$ administered intraperitoneally 15 mins before initiating the procedure, III) Ischemia reperfusion (IR): Bilateral clamping was done to initiate ischemia and maintained for 45 mins after that the kidneys subjected to 24 hrs of reperfusion, IV) Fisetin IR (F_IR): Similar to IR procedure. Fisetin $(20 \mathrm{mg} / \mathrm{kg})$ administered intraperitoneally 15 mins before initiating the procedure. V) Wortmannin IR (W_IR): Similar to IR procedure. Wortmannin $(0.015 \mathrm{mg} / \mathrm{kg})$ administered intraperitoneally 30 mins before initiating the procedure and $\mathrm{VI}$ ) Wortmannin Fisetin IR (W_F_IR): Wortmannin injected 15 mins before fisetin administration and proceeded with IR procedure.

\section{Biochemical Parameters}

Blood samples from all groups were collected before and after the surgery. Plasma was separated by

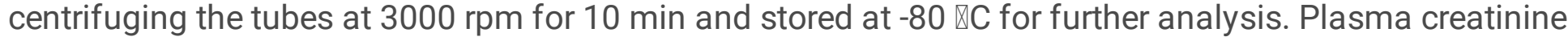
and BUN were estimated using the respective diagnostic kits purchased from Agappe diagnostics Ltd. (India).

Animals used for urine collection were housed in metabolic cages. The animals had free access to water during the entire experiment, which could last up to $24 \mathrm{~h}$ post-surgery. The urine was collected $24 \mathrm{hrs}$ after the surgery, and the total urine volume was determined. Creatinine and blood urea nitrogen (BUN) were measured in both plasma and urine using respective diagnostic kits from Agappe diagnostics Ltd (India). Creatinine, protein and blood urea nitrogen (BUN) were measured in the urine using diagnostic kits (Agappe, India) . 


\section{Glomerular Filtration rate}

eGFR calculation done using the equation by Pestel et al. (2007) (Pestel et al. 2007):

- Creatinine clearance $=(1000 \otimes$ urine volume $\otimes$ concentration of creatinine in urine $) /$ concentration of creatinine in serum

- $\mathrm{BUN}$ clearance $=$ (urine volume $\unrhd$ concentration of BUN in urine) $/$ concentration of BUN in serum

- $\mathrm{eGFR}=$ mean of (creatinine clearance, BUN clearance)

\section{Histopathology}

Immediate laparotomy was performed to collect both the kidneys after the reperfusion period. Isolated kidneys were cleaned off the extraneous tissue, weighed and rinsed with ice-cold normal saline. A section from both kidneys was fixed with $10 \% \mathrm{v} / \mathrm{v}$, embedded in paraffin, sectioned at $5 \mu \mathrm{m}$, and stained with hematoxylin and eosin for histopathological examination under a light microscope. Three kidney tissues per group were analyzed for histological changes.

\section{Apoptosis}

DNA was isolated using phenol chloroform isoamyl alcohol mixture as per standard protocol and sample $(1 \mu \mathrm{g} /$ well) was run on an agarose gel $(1.8 \%)$ for $2 \mathrm{~h}$. Laddering/smearing patterns representing DNA breaks due to apoptosis were evaluated along with standard DNA ladder and images were taken using Bio-Rad chemi Doc XRS system.

Additionally, renal tissue homogenate was prepared in Tris-Hcl buffer (pH-8) with EDTA (25 mM) and $\mathrm{NaCl}(400 \mathrm{mM})$. Activity of active caspase-3 was estimated spectrophotometrically using caspase-3 fluorogenic substrate (Sigma Aldrich, USA). Briefly the tissue homogenate was added to the buffer with substrate (HEPES $100 \mathrm{mM}$, EDTA $0.5 \mathrm{mM}$, DTT $5 \mathrm{mM}$, Glycerol 20\%, CHAPS $0.1 \%$ and substrate $25 \mu \mathrm{M}$ ) and the activity was monitored at ex/em $365 / 465 \mathrm{~nm}$ for $1 \mathrm{~h}$ at $370 \mathrm{C}$.

\section{Isolation of mitochondria}

The differential centrifugation technique was used to isolate rat kidney mitochondria according to the method described by Palmer et al (1977) (Palmer et al. 1977). Isolated mitochondrial function was analyzed as mentioned below.

\section{Antioxidant enzymes and lipid peroxidation}

The concentration of thiobarbituric acid reactive substances (TBARS) and glutathione (GSH) and activities of glutathione peroxidase (GPx), glutathione reductase (GR), superoxide dismutase (SOD) and catalase enzymes were measured in renal tissue and in mitochondrial samples as per the procedures described previously (Ansari et al. 2019). 


\section{Mitochondria analysis}

i) Activity of electron transport chain enzymes namely: Rotenone sensitive NADH-oxidoreductase (NQR), succinate decylubiquinone DCPIP reductase (SQR), ubiquinol cytochrome-c reductase (QCR) and cytochrome $\mathrm{c}$ oxidase (COX) analyzed spectrophotometric ally as per the procedure described previously (Priante et al. 2019).

ii) ATP level and ATP producing capacity: The ATPlite (Perkin Elmer) system was used for the measurement of ATP level in the isolated mitochondria of the all the groups based on the luminescence produced by the reaction of ATP with the substrates luciferase and a-luciferin. The ATP producing ability was evaluated in the presence of glutamate/malate $(5 / 2.5 \mathrm{mM})$ and succinate $(2.5 \mathrm{mM})$ energized medium and compared with the ATP level in the non energized conditions.

iii) Mitochondrial permeability: Ca2+-induced swelling was used to assess the opening of the mitochondrial permeability transition pore. Mitochondria were incubated at $30^{\circ} \mathrm{C}$ in swelling buffer containing $125 \mathrm{mM}$ sucrose, $50 \mathrm{mM} \mathrm{KCl}, 5 \mathrm{mM}$ HEPES, $2 \mathrm{mM} \mathrm{KH}_{2} \mathrm{PO}_{4}$ and $1 \mathrm{mM} \mathrm{MgCl}_{2}$ to assess nonenergized swelling. The absorbance was recorded at $540 \mathrm{~nm}$ for $10 \mathrm{~min}$. The swelling activity was reported as light scattering/mg protein at $540 \mathrm{~nm}$.

iv) Rhodamine 123 dye mediated transmembrane analysis: Mitochondria aliquots were centrifuged at $9000 \mathrm{~g}$ for $10 \mathrm{~min}$. Pellets were then suspended in fresh incubation buffer $(125 \mathrm{mM}$ Sucrose, $50 \mathrm{mMKCl}$, $5 \mathrm{mM} \mathrm{HEPES}, 2 \mathrm{mM} \mathrm{KH}_{2} \mathrm{PO}_{4}$ and $1 \mathrm{mM} \mathrm{MgCl}_{2}$ ) devoid of EDTA. $1.0 \mu \mathrm{M}$ of Rhodamine 123 was added and incubated at $37^{\circ}$ in a thermostatic bath for $15 \mathrm{~min}$ with gentle shaking. Mitochondrial pellets were separated by centrifugation at $9000 \mathrm{~g}$ for $10 \mathrm{~min}$ and the concentration of Rhodamine 123 was measured in the pellet as well as in supernatant at an excitation wavelength of $549 \mathrm{~nm}$ and emission wavelength of $574 \mathrm{~nm}$. Membrane potentials (negative inside) were calculated by the Nernst equation: $\Delta \psi \mathrm{m}=59 * \log$ [(Rh 123)in/(Rh 123)out].

v) Gene expression: The expression for the mitochondrial encoded $\mathrm{Nd} 1$, and nuclear-encoded $\beta$ actin, Tfam, Polg, Pgc 1a, Fis 1, Mff, Dnm 1, Mfn 1, Mfn 2, Opa 1, Pink 1, Parkin, and Optn was analyzed using qPCR (ABI7500, Thermo Scientific, USA) in rat kidney samples. The primer details are presented in Table 1. Nd 1 expression analyzed in both cellular DNA and CDNA whereas other gene expressions estimated in cDNA and normalized with $\beta$ actin in respective samples. The DNA isolation is done using phenolchloroform-isoamyl alcohol method according to manufacturer's instructions (Himedia, Mumbai). The mRNA extraction was carried out using TRIzol reagent (15596026, Thermo Scientific, USA) as per the instructions, and gene expression was quantified using Sybr green chemistry (F415, Thermo Scientific, USA). The expression of genes was calculated as per the procedure of Livak and Schmittgen ( Livak and Schmittgen 2001). Primer sequence of the genes are presented in Table 1.

\section{Statistical Analysis}


All data are expressed as mean \pm standard deviation of the mean (SD). Intergroup comparisons were performed by using a one-way analysis of variance, followed by post hoc Dunnet's test was employed to determine the difference among the groups, $\mathrm{P}<0.05$ was considered statistically significant.

\section{Results}

\section{The effects of fisetin on eGFR, plasma BUN, creatinine, and urinary albumin/creatinine ratio in rats subjected to renal ischemia reperfusion injury.}

The effect of fisetin on the renal physiological function and corresponding renal markers were measured and the results are given in figure 1 . According to figure $1 \mathrm{a}, \mathrm{IR}$ induced a fall in the eGFR from the sham group (S- $15.91 \mathrm{ml} / \mathrm{min}, \mathrm{IR}-0.35 \mathrm{ml} / \mathrm{min}$ ). The plasma level of BUN (S- $18.34 \mathrm{mg} / \mathrm{dl}$, IR- $111.83 \mathrm{mg} / \mathrm{dl}$ ), creatinine (S- $0.69 \mathrm{mg} / \mathrm{dl}, \mathrm{IR}-2.20 \mathrm{mg} / \mathrm{dl}$ ) and urinary albumin/creatinine ratio (Al/Cr-S-0.46, IR-6.09) were significantly declined from the normal range in IR group (Figure 1b-1d).

Fisetin pre-treatment prior to IR protocol protected the kidney from damage and improved the glomerular function significantly $(p<0.05)$ from the IR (IR- $\left.0.35 \mathrm{ml} / \mathrm{min}, \mathrm{F}_{-} \mid \mathrm{R}-9.03 \mathrm{ml} / \mathrm{min}\right)$. The injury markers exhibited a marked reduction in their level in fisetin pretreated renal tissues from the IR group (F-IR: $\mathrm{Cr}$ $0.92 \mathrm{mg} / \mathrm{dl}, \mathrm{BUN}-81.03 \mathrm{mg} / \mathrm{dl}, \mathrm{Al} / \mathrm{Cr}-2.16)$.

The renal injury markers in the blood exhibited a marked reduction in the level in fisetin pretreated renal tissues from the IR group (F-IR: Cr- $0.92 \mathrm{mg} / \mathrm{dl}$ vs $2.20 \mathrm{mg} / \mathrm{dl}, \mathrm{BUN}-81.03 \mathrm{mg} / \mathrm{dl}$ vs $111.83 \mathrm{mg} / \mathrm{dl}, \mathrm{Al} / \mathrm{Cr}-$ 2.16 vs 6.09 from IR group).

\section{Effect of fisetin on renal injury in rats subjected to renal ischemia reperfusion.}

Histopathological analysis of the renal tissue from different experimental groups reveals normal architecture in the sham group, which was significantly altered in the IR tissue. Compared with the sham (Figure 1e), the kidneys from the IR group rats (Figure 1f) showed severe glomerular congestion, tubular dilatation, tubular degeneration with vacuolization in proximal tubules and cast formation. The pathological changes in the renal tissues from IR groups were minimal with prior treatment of fisetin (Figure $1 \mathrm{~h}$ ) confirming the protective effect of fisetin. Accordingly, the characteristic histological changes observed in F_IR were decrease in renal congestion and tubular dilatation and minimal tubular cast formation indicated a reduced injury (Figure 1i).

\section{Fisetin attenuates the IR induced apoptosis in rats subjected to renal ischemia reperfusion injury.}

Apoptotic renal injury was assessed by measuring the caspase 3 activities and visualizing the fragmented DNA in agarose gel (Figure $1 \mathrm{j}$ and $1 \mathrm{k}$ ). Caspase 3 activity was high in IR tissue by $27 \%$ from the sham tissue. Fragmented DNA was visible in the electrogram of IR tissue which was absent in the sham group. However, compared with the IR, fisetin pre-treated IR renal tissue exhibited significantly low apoptotic injury evident by the reduced fragmentation and decline in caspase 3 activity by 19\% (Figure $1 \mathrm{k})$. 


\section{Fisetin reduced the oxidative stress associated with renal ischemia reperfusion injury.}

Figure 2 shows the renal lipid peroxidation level and antioxidant enzyme activities in the tissues of different experimental groups. Upon reperfusion, the renal tissues exhibited increased concentration of TBARS (in nM MDA/mg tissue, S- 2.00, IR- 3.41) and correspondingly decreased level of GSH/GSSG (S0.43 , IR- 0.27). The antioxidant enzymes like SOD (in U/mg protein, S-5.8, IR-4.56), catalase ( $\mathrm{mU} / \mathrm{mg}$ protein, S-12.8, IR- 6.03) and GPx ( $\mu \mathrm{M}$ GSH/mg protein, S- 0.94, IR- 0.65) were declined in their activities. Preconditioning the animal with fisetin showed a significant decline in the level of TBARS (IR- 3.41, F_IR2.74) and improved the endogenous antioxidant GSH/GSSG level (IR-0.27, F_IR-0.34) compared with the IR group. The antioxidant enzyme activities (SOD, catalase, GPx, and GR) were improved in F_IR (Figure $2 c-f)$ group than IR.

Figure 3 shows the lipid peroxidation and antioxidant status in the renal mitochondria. Lipid peroxidation measured in terms of TBARS was high in IR group (11\% higher than in the homogenate). The corresponding decline in reduced glutathione (GSH) was prominent in IR tissue (31\% higher than in homogenate). Similar patterns of changes were observed in the antioxidant enzymes of mitochondrial fraction from IR tissue. Fisetin pretreatment significantly reduced TBARS (42\%) and increased GSH/GSSG (18\%) level in the mitochondria from the IR group animals. Activities of antioxidant enzymes like catalase (63\%), SOD (35\%), and GPx (20\%) were also improved significantly from the IR group.

\section{Fisetin improved the mitochondrial bio-energetic functions in rats subjected to ischemia reperfusion injury.}

The quality of mitochondria in the renal tissue is a critical factor that regulate renal function. The different mitochondria functions like bioenergetics (Figure 4) and its regulatory events such as copy number (Figure 5), mitophagy, mitofusion, and mitofission (Figure 6) were evaluated to assess the renal mitochondrial quality.

Bioenergetic potential of renal mitochondria was measured via ETC enzyme activities like NQR, SQR, QCR and COX along with ATP level (Figure 4). Animals subjected to IR exhibited low enzyme activities in NQR, SQR, QCR and COX by $41 \%, 43 \%, 50 \%$, and $82 \%$ respectively from the sham. ATP level in IR tissue was significantly low (18\%) from the sham. However, Fisetin treatment in tissue subjected to IR improved the mitochondrial ETC enzyme activities of NQR (25\%), SQR (25\%), QCR (39\%), COX (71\%), and ATP level (22\%) from the IR control (Figure 4a-d and 7a).

To assess if the mitochondrial copy number plays a significant role in the reduced functional activity, we evaluated the mitochondrial copy number in the rat renal tissues from all the experimental groups and noted a significant $(p<0.05)$ reduction in the copy number in IR treated animal which was improved by the pre-treatment of the animals with fisetin (Figure 5a).

Further analysis of the expression level of PGC-1a (Figure 5b), which plays a critical role in the regulation of mitochondrial biogenesis and respiratory function (bioenergetics) showed $62 \%(p<0.05)$ decline in IR 
kidney. Fisetin pretreatment was found to improve the PGC-1a expression by $63 \%$ from the IR group confirming the mitochondrial biogenetic potential of the drug. However, fisetin found to exert minimum impact on the mitochondrial transcription regulation factor $\mathrm{A}(\mathrm{Tfam})$ and mitochondrial DNA polymerase $Y$ (POLG) which regulates the mitochondrial encoded gene expressions, where 13 mitochondrial encoded genes influenced the activity of bio-energetic enzymes (Figure $5 c-5 d$ ).

\section{Fisetin improved renal mitochondrial dynamics and mitophagy in rats subjected to ischemia reperfusion injury.}

The damage to mitochondria associated with IR injury can disrupt the balance of the dynamic processes controlling mitochondrial fusion and fission. In order to analyze the same we estimated the gene expression of mitochondrial fission and fusion genes in different experimental groups and the results are given in figure 6. We observed an augmented expression of mitochondrial fission genes Mff, Fis 1 and Dmn 1 by $1.66,1.17$ and 2.23 folds respectively in the IR treated groups compared with sham (Figure 6a6c). Treatment with fisetin prior the IR reduced the mitochondrial fission (Dmn 1 expressed in Fold change: IR-2.23 F_IR-1.21, Fis 1: IR-1.17 F_IR-0.35, Mff: IR-1.66 F_IR-1.14). Furthermore the mRNA expression of Mfn1 gene, which codes for the principal GTP-dependent membrane tethering protein for mitochondrial fusion exhibited a significant $(p<0.05)$ increase in mitchondrial fusion gene expression due to in IR (S-1.00, IR-1.57) group animals. However, mfn1 expression was further increased in F_IR group (IR-1.57, F_IR-1.92).

To determine the effect of fisetin on mitophagy -an important event that maintain the mitochondrial quality was evaluated by measuring the gene expression of the key regulatory molecules such as Pink, Parkin and Optn (Figure 6f- 6h). Among the three genes, Pink and Parkin showed significant elevation (3.7 fold) in the gene expression in response to IR induction in the sham control. On the other hand, compared to sham all the three genes showed significantly higher expression in F_IR. Two out of three genes were significantly expressed in F_IR group compared to the IR control group (Parkin: IR- 1.25, F_IR- 1.98 and Optn: IR-1.00, F_IR-1.28). In general, fisetin pretreatment prior to IR challenged improved the mitochondrial function and maintained the mitochondrial homeostasis and quality.

\section{Fisetin improved the mitochondrial ATP producing capacity, and attenuated swelling and mPTP opening in rats subjected to ischemia reperfusion injury.}

In order to assess if alterations in the electron transport chain affected the ATP machinery in renal tissue we estimated the ATP producing capacity of isolated mitochondria in different energised conditions (Glutamate-Malate/Succinate) and non-energised conditions. After incubating the mitochondria in the respective respiratory buffers for 10 minutes, ATP content was measured by using ATPlite kit and the results are presented in Figure 7 (a- $\mathrm{c}$ ). The IR significantly compromised the ATP producing capacity of the isolated renal mitochondria in both energized and non-energized conditions. However, isolated renal mitochondria from the fisetin pretreatment group retain the ATP producing capacity significantly. We also noted another interesting observation where the ATP level showed a striking increase in the fisetin control group than the sham control. 
The corresponding mitochondrial membrane potential, that determine the oxidative phosphorylation, in different energized medium did not show significant changes across the experimental groups (Figure 7d7f). However, mitochondrial swelling was high in IR group in both energized and non-energized conditions, which was improved substantially in fisetin treated IR group (Figure 7g-7i).

\section{Fisetin's protective ability towards renal IR injury is independent of PI3K/Akt signalling pathway}

In order to assess the role of PI3K/Akt pathway in the renal protection mediated by fisetin, we used an inhibitor based study. The study results presented in figure 8 shows that the IR induced renal injury was found to be intensified in presence of Wortmannin, a PI3K inhibitor confirming the role of PI3K in renal IR protection. However, the protective ability of fisetin towards IR was retained even in the presence of Wortmannin indicated that the fisetin mediated renal protection towards renal IR injury is independent of $\mathrm{PI} 3 \mathrm{~K} /$ Akt signalling pathway.

\section{Discussion}

Renal ischemia reperfusion injury contribute significantly to the development of acute kidney injury that continuously associated with high morbidity and mortality rate (Basile et al. 2012). Early reports suggests that ischemia reperfusion injury in rat heart can be effectively ameliorated by fisetin administration (Shanmugam et al. 2018). Pathophysiology of IR in heart and kidney are different due to difference in the composition of cell types (Kisseleva and Brenner 2008). However, therapeutic efficacy of fisetin in attenuating IR injury in renal tissue is yet to be explored. The important findings of the present study are as follows (1) IR induced reduction in eGFR and increased plasma BUN, creatinine and urinary $\mathrm{Al} / \mathrm{Cr}$ ratio was reversed by fisetin and thereby preserved renal physiology from the impact of ischemia reperfusion. (2) IR increased oxidative stress and mitochondrial dysfunction were attenuated by fisetin (3) Mitochondrial copy number reduction in the tissues challenged to ischemia reperfusion were restored by fisetin (4) IR associated decline in the mitochondrial quality in renal tissues were protected by fisetin pretreatment via reversing the gene expression of Pgc 1a, reducing mitochondrial fission (Mff, Fis, and Dnm 1), increasing mitochondrial fusion (Mfn 1) and mitophagy (Parkin and Optn) (5) The protective effect of fisetin against renal ischemia reperfusion injury is independent of PI3K/Akt signaling pathway.

Ischemia reperfusion injury compromised the renal function by altering the renal hemodynamics and thereby promoting the development of AKI (Basile et al. 2012). In the present study, the IR associated deterioration of renal function was assessed by measuring the efficiency of eGFR and the levels of plasma BUN, creatinine and urinary albumin to creatinine ratio (Figure 1a- 1d). The improvement of these parameters in fisetin treated IR challenged kidney tissue confirmed the ability of the compound to render renal protection against IR. Renal cell death can contribute to the deteriorated IR associated renal insufficiency (Priante et al. 2019). Hence, we evaluated the ultra-structure of kidney in all experimental groups. Accordingly, we found severe renal injury in IR group and this injury was significantly reduced with fisetin pretreatment (Figure 1e-1i). Fisetin was reported to attenuate IR associated apoptotic cell death in heart, brain and liver (Shanmugam et al. 2018; Li et al. 2021; Zhang and Cui 2021). In 
accordance with this finding, the present study demonstrated prominent decline in apoptotic injury in F_IR group (Figure 1j-1k).

Renal proximal tubule is the primary target of ischemia reperfusion injury and involved in the subsequent progression of kidney diseases. This may be due to the presence of large number of mitochondria in PCT (Chevalier 2016). The IR injury in any organ is developed when there is an imbalance between energy supply and demand, which is regulated by mitochondria (Kalogeris et al. 2012). In the present study, we noted deteriorated mitochondrial function in IR challenged renal tissue. Importantly, most of the IR associated mitochondrial specific changes were improved in renal tissue that were priorly treated with fisetin. The overall function of mitochondria can be assessed by measuring different mitochondrial events like bioenergetics, mitophagy, mitofusion and mitochondrial copy number, collectively called mitochondrial quality control. Recent evidence emphasizes the significance of mitochondrial quality control as a molecular target in the management of ischemia reperfusion.

Previous studies reported the role of the master regulatory gene- Pgc 1a, in the energy metabolism (Liang and Ward 2006). Pagel-Langenickel identified Pgc $1 \mathrm{a}$ as a bidirectional regulatory link integrating insulinsignaling and mitochondrial homeostasis in skeletal muscle (Pagel-Langenickel et al. 2008). In the present study, expression of Pgc 1a was significantly low in IR tissues (60\% from sham) was recovered to near normal level by fisetin treatment (fig). The results are in corroboration with our previous results reported in the heart (Shanmugam et al. 2018). Consistent with this observation, we found the improvement of bioenergetic function in fisetin treated renal tissue where NQR, SQR, QCR and COX were significantly elevated from the IR control animal (Figure 4). In addition, ATP level in the tissue was augmented with fisetin treatment (Figure 7).

Pgc $1 \mathrm{a}$ is not only a key regulator of energy metabolism but also control mitochondrial biogenesis (Liang and Ward 2006). Flavonoids belonging to all classes have been found to stimulate mitochondrial biogenesis in both in vitro and in vivo by activating PGC 1a (Pagel-Langenickel et al. 2008; Kicinska and Jarmuszkiewicz 2020). In this direction we found significant improvement in mitochondrial fusion genes like Mfn 1 in fisetin treated renal tissue that may mitigate the stress in mitochondria by undergoing fusion with healthy mitochondria (Figure 6). Previous studies have showed that Pgc 1a regulate the expression of mitochondrial antioxidant enzymes (Lu et al. 2010). In the present study we measured the oxidative stress experienced by the mitochondria and tissue homogenate and the results complemented with Pgc 1a where an improved antioxidant status in renal tissues from IR were observed in rats pretreated with fisetin (Figure 2 and 3).

Literature suggest that when the mitochondria experienced low membrane potential, it triggers mitochondrial events that favors fission that in turn enhances mitophagy to eliminate the dysfunctional mitochondria (Twig and Shirihai 2011). The mitochondrial fusion genes were significantly elevated in renal IR tissue with subsequent upregulation of mitophagic genes (Figure 6). Fisetin pretreatment reduced the expression of mitochondrial fission genes and increased the expression of fusion and phagy genes. Similar effects were shown by quercetin, another dietary flavonoid that is structurally similar to fisetin, in 
vascular smooth muscle cells (Cui et al. 2017). The overall mitochondrial functional improvement by fisetin treatment was reflected in restoring mitochondrial DNA copy number (Figure 5).

In order to reconfirm the fisetin mediated mitochondrial protection, we isolated the mitochondria from different experimental groups and incubated the organelle in energized (GM/ succinate) and nonenergized medium before measuring the ATP producing capacity and related mitochondrial membrane potential and swelling behavior (Figure 7). Accordingly the results given in figure 7a-7c suggests high ATP producing capacity where in both fisetin treated group in all conditions. This observation was in lane with previous finding that suggest the activation of complex 1 by polyphenolic compound resveratrol (Gueguen et al. 2015). Structural similarities of fisetin with resveratrol predict the ability of fisetin to donate electrons to ETC, thereby promote the generation of proton motive force (PMF) that may culminate in ATP production.

Fisetin was reported to render cardioprotection against IR via activating PI3K signalling pathway (Shanmugam et al. 2021). Contrary to this finding the present study result did not support PI3K mediated protection in renal IR, where loss of function of PI3K by wortmannin did not show any significant decline in renal protection mediated by fisetin (Figure 8).

\section{Conclusion}

The present study shows that IR challenge compromised renal function (reduced eGFR and increased kidney injury markers), deteriorated mitochondrial function along with low mitochondrial quality. These changes were successfully reverted by the fisetin treatment and protected the kidney from renal IR injury.

\section{Abbreviations}

IR-Ischemia reperfusion

AKI- Acute kidney injury

eGFR- Estimated glomerular filtration rate

ATP- Adenosine triphosphate

Al/Cr- urinary albumin/creatinine ratio

PI3k-phosphoinositide 3-kinases

Akt- Protein kinase B

GSK3 $\beta$ - Glycogen synthase kinase 3 beta 
BUN-Blood urea nitrogen

MDA- Malondialdehyde

GSH- Reduced glutathione

GSSG- Oxidised glutathione

GPx- Glutathione peroxidase

SOD-Superoxide dismutase

GR- Glutathione reductase

NQR- NADH-oxidoreductase

SQR- succinate-coenzyme $Q$ reductase

QCR-QH2

cytochrome c oxidoreductase

cOX-cytochrome c oxidase

Pgc 1a-Peroxisome proliferator-activated receptor-gamma coactivator

Tfam- transcription regulation factor $A$

Polg- mitochondrial DNA polymerasey

Dnm 1- Dynamin-1

Fis 1- Mitochondrial fission protein 1

Mff- Mitochondrial fission factor

Mfn1-Mitofusin 1

Mfn 2- Mitofusin 2

Pink-PTEN-induced kinase 1 
Optn- Optineurin.

\section{Declarations}

\section{Funding}

The authors sincerely thank Department of Science and Technology, India for supporting this research through grant-in-aid (EMR/2017/000669). Ms. Priyanka N P was supported by Council of Scientific and Industrial Research, India (CSIR) fellowship grant (09/1095/(0040)/2018-EMR-1).

\section{Conflict of Interest}

The authors declare that the research was conducted in the absence of any potential conflict of interest.

\section{Data availability statement}

The datasets generated and analyzed during the current study are submitted as supplementary material.

\section{Ethics approval}

All the animal experiments carried out are in compliance with the guidelines of Committee for the Purpose of Control and Supervision of Experiments on Animals (CPCSEA) and approved by the Institutional animal ethical committee (552/SASTRA/IAEC/RPP).

\section{Author Contributions}

PNP has processed the experimental data, performed analysis, drafted the manuscript, designed figures \& tables, and compiled the literature sources. GAK has contributed to the design and implementation of the research, to the interpretation of the results, and to the writing of the manuscript. The authors declare that all data were generated in-house and that no paper mill was used.

\section{Acknowledgments}

The authors thank Dr. David C Raj for his extensive help in carrying out the animal surgeries. We thank SASTRA Deemed University for providing all the needed infrastructure and facilities.

\section{References}

1. Basile DP, Anderson MD, Sutton TA (2012) Pathophysiology of acute kidney injury. Compr Physiol 2(2):1303-1353. doi:10.1002/cphy.c110041

2. Ding X, Cheng Z, Qian Q (2017) Intravenous Fluids and Acute Kidney Injury. Blood Purif 43(1-3):163172. doi:10.1159/000452702 
3. Doyle JF, Forni LG: Acute kidney injury: short-term and long-term effects. Crit Care 20(1):188 (2016) Published 2016 Jul 4. doi:10.1186/s13054-016-1353-y

4. Jo SK, Rosner MH, Okusa MD (2007) Pharmacologic treatment of acute kidney injury: why drugs haven't worked and what is on the horizon. Clin J Am Soc Nephrol 2(2):356-365. doi:10.2215/CJN.03280906

5. Vargas F, Romecín P, García-Guillén Al, Wangesteen R, Vargas-Tendero P, Paredes MD, Atucha NM, García-Estañ J (2018) Flavonoids in Kidney Health and Disease. Front Physiol 9:394. doi:10.3389/fphys.2018.00394

6. Panche AN, Diwan AD, Chandra SR (2016) Flavonoids: an overview. J Nutr Sci 5:e47. doi:10.1017/jns.2016.41

7. Arab HH, Mohamed WR, Barakat BM (2016) Arafa el-SA: Tangeretin attenuates cisplatin-induced renal injury in rats: Impact on the inflammatory cascade and oxidative perturbations. Chem Biol Interact 258:205-213. doi:10.1016/j.cbi.2016.09.008

8. Bai S, Huang ZG, Chen L, Wang JT, Ding BP (2013) Effects of felodipine combined with puerarin on ACE2-Ang (1-7)-Mas axis in renovascular hypertensive rat. Regul Pept 184:54-61. doi:10.1016/j.regpep.2013.03.005

9. Khan N, Syed DN, Ahmad N, Mukhtar H (2013) Fisetin: a dietary antioxidant for health promotion. Antioxid Redox Signal 19(2):151-162. doi:10.1089/ars.2012.4901

10. Antika LD, Dewi RM (2021) Pharmacological aspects of fisetin. Asian Pac J Trop Biomed 11(1):1

11. Touil YS, Auzeil N, Boulinguez F, Saighi H, Regazzetti A, Scherman D, Chabot GG (2011) Fisetin disposition and metabolism in mice: Identification of geraldol as an active metabolite. Biochem Pharmacol 82(11):1731-1739. doi:10.1016/j.bcp.2011.07.097

12. Prasath GS, Subramanian SP (2011) Modulatory effects of fisetin, a bioflavonoid, on hyperglycemia by attenuating the key enzymes of carbohydrate metabolism in hepatic and renal tissues in streptozotocin-induced diabetic rats. Eur J Pharmacol 668(3):492-496. doi:10.1016/j.ejphar.2011.07.021

13. Shanmugam K, Ravindran S, Kurian GA, Rajesh M (2018) Fisetin Confers Cardioprotection against Myocardial Ischemia Reperfusion Injury by Suppressing Mitochondrial Oxidative Stress and Mitochondrial Dysfunction and Inhibiting Glycogen Synthase Kinase $3 \beta$ Activity. Oxid Med Cell Longev 2018:9173436. doi:10.1155/2018/9173436

14. Li Z, Wang Y, Zhang Y, Wang X, Gao B, Li Y, Li R, Wang J: Protective Effects of Fisetin on Hepatic Ischemia-reperfusion Injury Through Alleviation of Apoptosis and Oxidative Stress. Arch Med Res 52(2):163-173, 202. doi: 10.1016/j.arcmed.2020.10.009

15. Zhang P, Cui J: Neuroprotective Effect of Fisetin Against the Cerebral Ischemia-Reperfusion Damage via Suppression of Oxidative Stress and Inflammatory Parameters [published online ahead of print, 2021 Feb 22]. Inflammation 10.1007/s10753-021-01434-x, 2021. doi:10.1007/s10753-021-01434-x

16. Kisseleva T, Brenner DA (2008) Fibrogenesis of parenchymal organs. Proc Am Thorac Soc 5(3):338342. doi:10.1513/pats.200711-168DR 
17. Pestel S, Krzykalla V, Weckesser G (2007) Measurement of glomerular filtration rate in the conscious rat. J Pharmacol Toxicol Methods 56(3):277-289. doi:10.1016/j.vascn.2007.03.001

18. Palmer JW, Tandler B, Hoppel CL (1977) Biochemical properties of subsarcolemmal and interfibrillar mitochondria isolated from rat cardiac muscle. J Biol Chem 252(23):8731-8739

19. Ansari M, Gopalakrishnan S, Kurian GA (2019) Streptozotocin-induced type II diabetic rat administered with nonobesogenic high-fat diet is highly susceptible to myocardial ischemiareperfusion injury: An insight into the function of mitochondria. J Cell Physiol 234(4):4104-4114

20. Livak KJ, Schmittgen TD: Analysis of relative gene expression data using real-time quantitative PCR and the $2-\Delta \Delta$ CT method. Methods 1;25(4):402-8, 2001

21. Priante G, Gianesello L, Ceol M, Del Prete D, Anglani F: Cell Death in the Kidney. Int J Mol Sci 20(14):3598 (2019) Published 2019 Jul 23. doi:10.3390/ijms20143598

22. Chevalier RL (2016) The proximal tubule is the primary target of injury and progression of kidney disease: role of the glomerulotubular junction. Am J Physiol Renal Physiol 311(1):F145-F161. doi:10.1152/ajprenal.00164.2016

23. Kalogeris T, Baines CP, Krenz M, Korthuis RJ (2012) Cell biology of ischemia/reperfusion injury. Int Rev Cell Mol Biol 298:229-317. doi:10.1016/B978-0-12-394309-5.00006-7

24. Liang H, Ward WF (2006) PGC-1alpha: a key regulator of energy metabolism. Adv Physiol Educ 30(4):145-151. doi:10.1152/advan.00052.2006

25. Pagel-Langenickel I, Bao J, Joseph JJ, Schwartz DR, Mantell BS, Xu X, Raghavachari N, Sack MN (2008) PGC-1alpha integrates insulin signaling, mitochondrial regulation, and bioenergetic function in skeletal muscle. J Biol Chem 283(33):22464-22472. doi:10.1074/jbc.M800842200

26. Kicinska A, Jarmuszkiewicz W (2020) Flavonoids and Mitochondria: Activation of Cytoprotective Pathways? Molecules 25(13):3060. doi:10.3390/molecules25133060

27. Lu Z, Xu X, Hu X, Fassett J, Zhu G, Tao Y, Li J, Huang Y, Zhang P, Zhao B, Chen Y (2010) PGC-1 alpha regulates expression of myocardial mitochondrial antioxidants and myocardial oxidative stress after chronic systolic overload. Antioxid Redox Signal 13(7):1011-1022. doi:10.1089/ars.2009.2940

28. Twig G, Shirihai OS (2011) The interplay between mitochondrial dynamics and mitophagy. Antioxid Redox Signal 14(10):1939-1951. doi:10.1089/ars.2010.3779

29. Cui L, Li Z, Chang X, Cong G, Hao L (2017) Quercetin attenuates vascular calcification by inhibiting oxidative stress and mitochondrial fission. Vascul Pharmacol 88:21-29.

doi:10.1016/j.vph.2016.11.006

30. Gueguen N, Desquiret-Dumas V, Leman G, Chupin S, Baron S, Nivet-Antoine V, Vessières E, Ayer A, Henrion D, Lenaers G, Reynier P, Procaccio V (2015) Resveratrol Directly Binds to Mitochondrial Complex I and Increases Oxidative Stress in Brain Mitochondria of Aged Mice. PLoS One 18(12):e0144290. doi:10.1371/journal.pone.0144290 10)

31. Shanmugam K, Boovarahan SR, Prem P, Sivakumar B, Kurian GA (2021) Fisetin Attenuates Myocardial Ischemia-Reperfusion Injury by Activating the Reperfusion Injury Salvage Kinase (RISK) Signaling Pathway. Front Pharmacol 12:566470. doi:10.3389/fphar.2021.566470 


\section{Tables}

Table 1 is not available with this version.

\section{Figures}
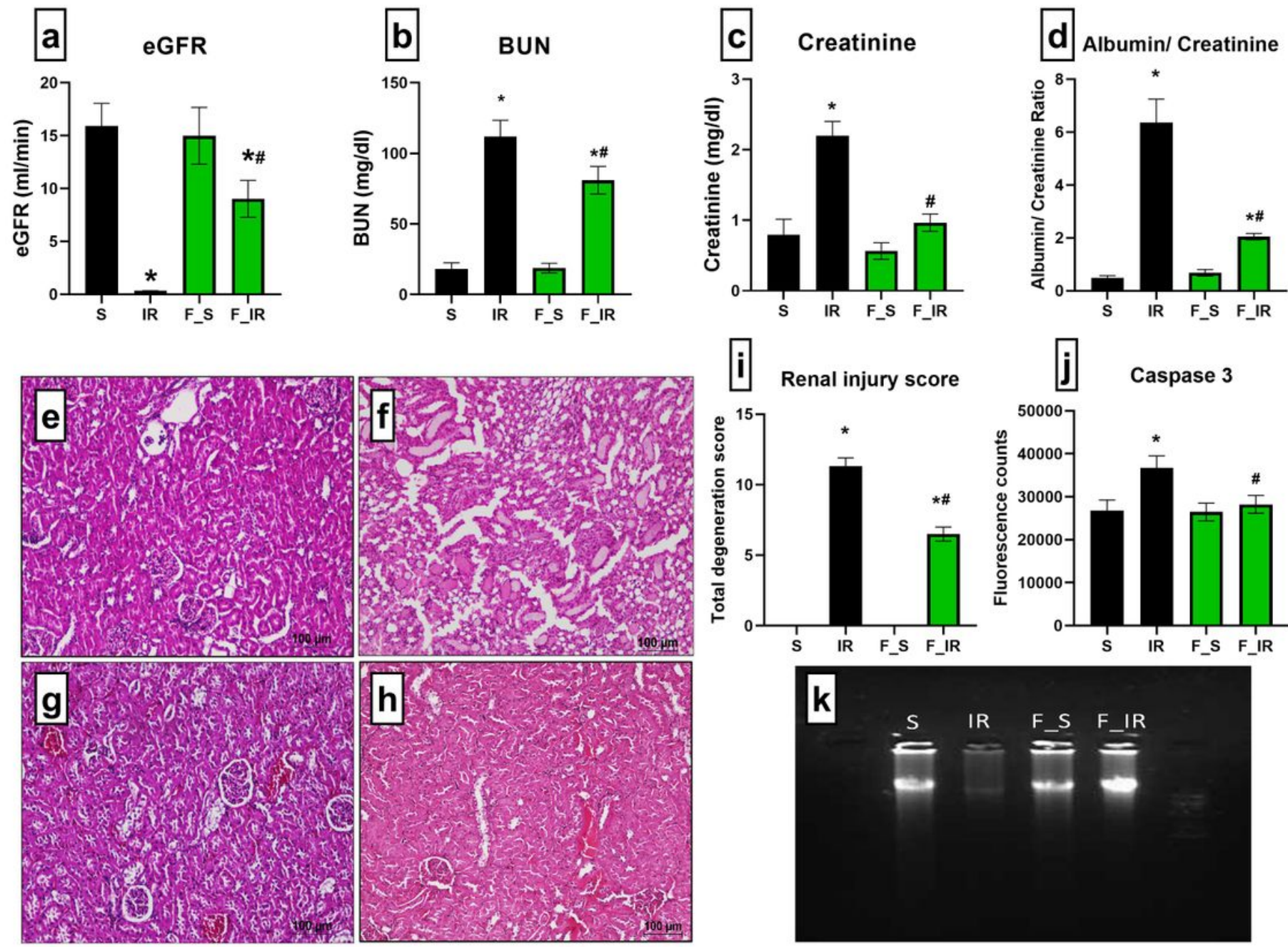

\section{Figure 1}

Renal physiology and injury analysis of sham and fisetin treated rats subjected to IR injury. Renal functional markers (a) eGFR, (b) Plasma creatinine, (c) Plasma BUN, and (d) Urinary albumin/Creatinine ratio. H\&E stained severe necrotic derangement images for (e) S, (f) IR, (g) F_S and (h) F_IR and (i) Renal injury score from $\mathrm{H}$ and $\mathrm{E}$ staining. The images are presented at 10x magnification. Renal cell death analysis by (j) caspase activity and (k) DNA fragmantation. ${ }^{*} p<0.05$ vs $S, \# p<0.05$ vs IR. The data are presented as mean $\pm S D(n=6 /$ group). S-Sham, IR-Ischemia-reperfusion, F_S- Fisetin sham, F_IR- Fisetin ischemia-reperfusion. 


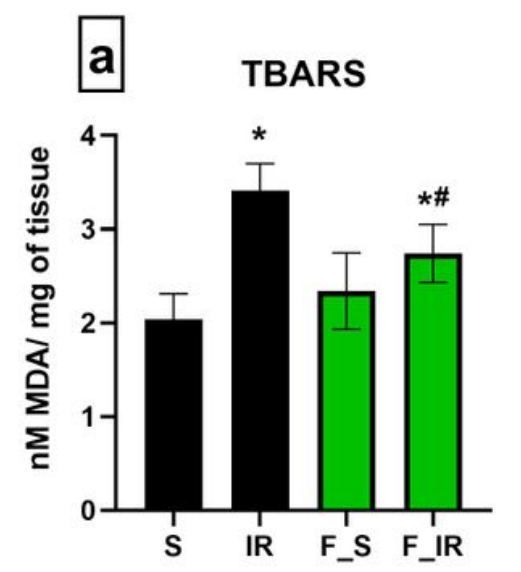

b GSH/GSSG

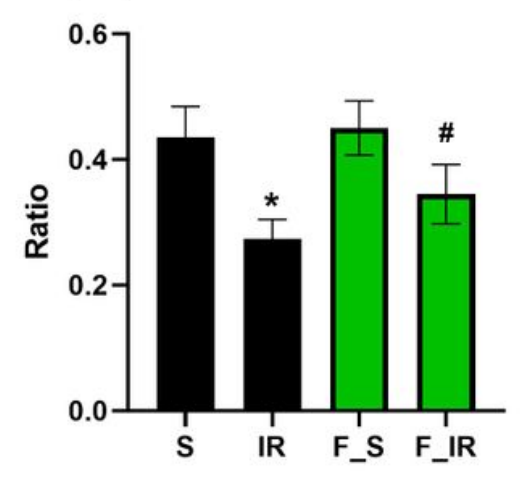

e

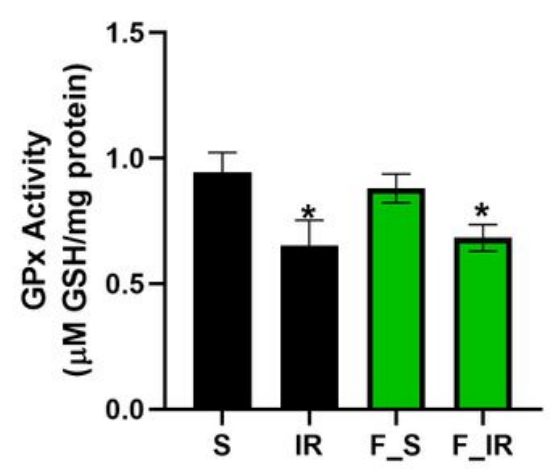

C SOD

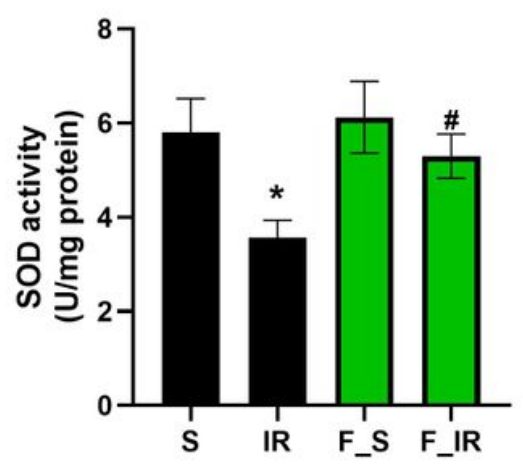

f

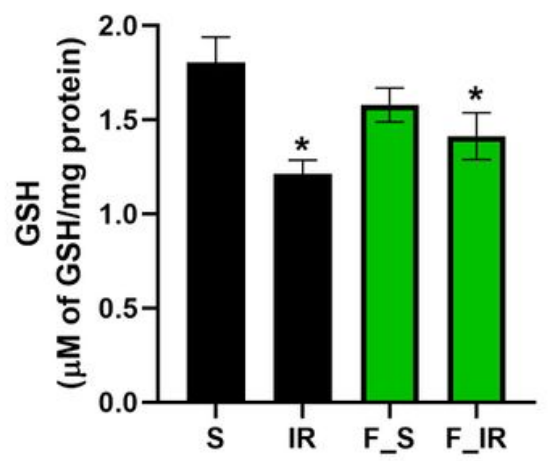

Figure 2

Oxidative stress analysis and antioxidants levels in the renal homogenate of sham and fisetin treated rats subjected to IR injury. (a) Shows lipid peroxidation measured by TBARS, (b) GSH/GSSG ratio, (c) SOD activity, (d) Catalase activity, (e) represents GPx activity and f) Total GSH. ${ }^{*} p<0.05$ vs $S, \# p<0.05$ vs IR. The data are presented as mean \pm SD ( $n=6 /$ group). S-Sham, IR-Ischemia-reperfusion, F_S- Fisetin sham, F_IR- Fisetin ischemia-reperfusion. 


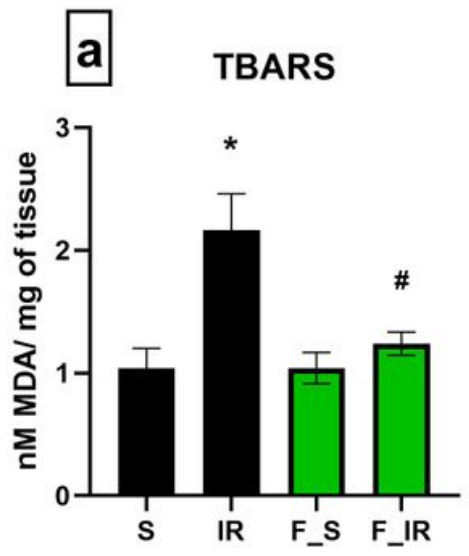

b GSH/GSSG

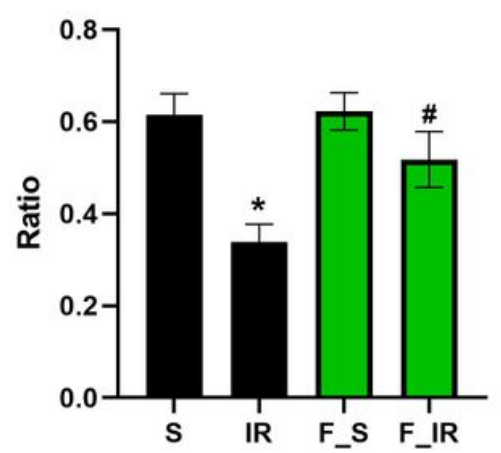

e

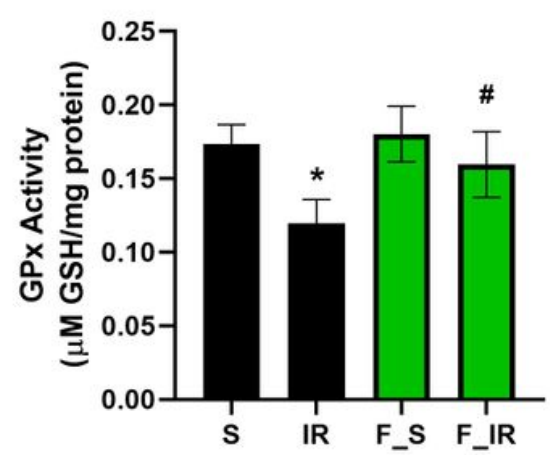

c SOD

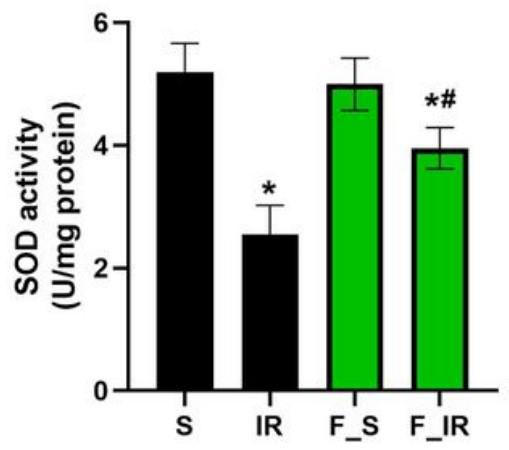

f

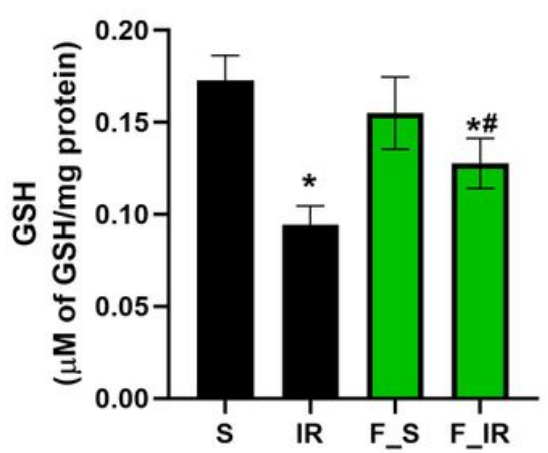

Figure 3

Oxidative stress analysis and antioxidants levels in the renal mitochondrial fraction of sham and fisetin treated rats subjected to IR injury. (a) Shows lipid peroxidation measured by TBARS, (b) GSH/GSSG ratio, (c) SOD activity, (d) Catalase activity, (e) represents GPx activity and f) Total GSH. * $p<0.05$ vs $\mathrm{S}, \# p<0.05$ vs IR. The data are presented as mean \pm SD ( $n=6$ /group). S-Sham, IR-Ischemia-reperfusion, F_S- Fisetin sham, F_IR- Fisetin ischemia-reperfusion. 

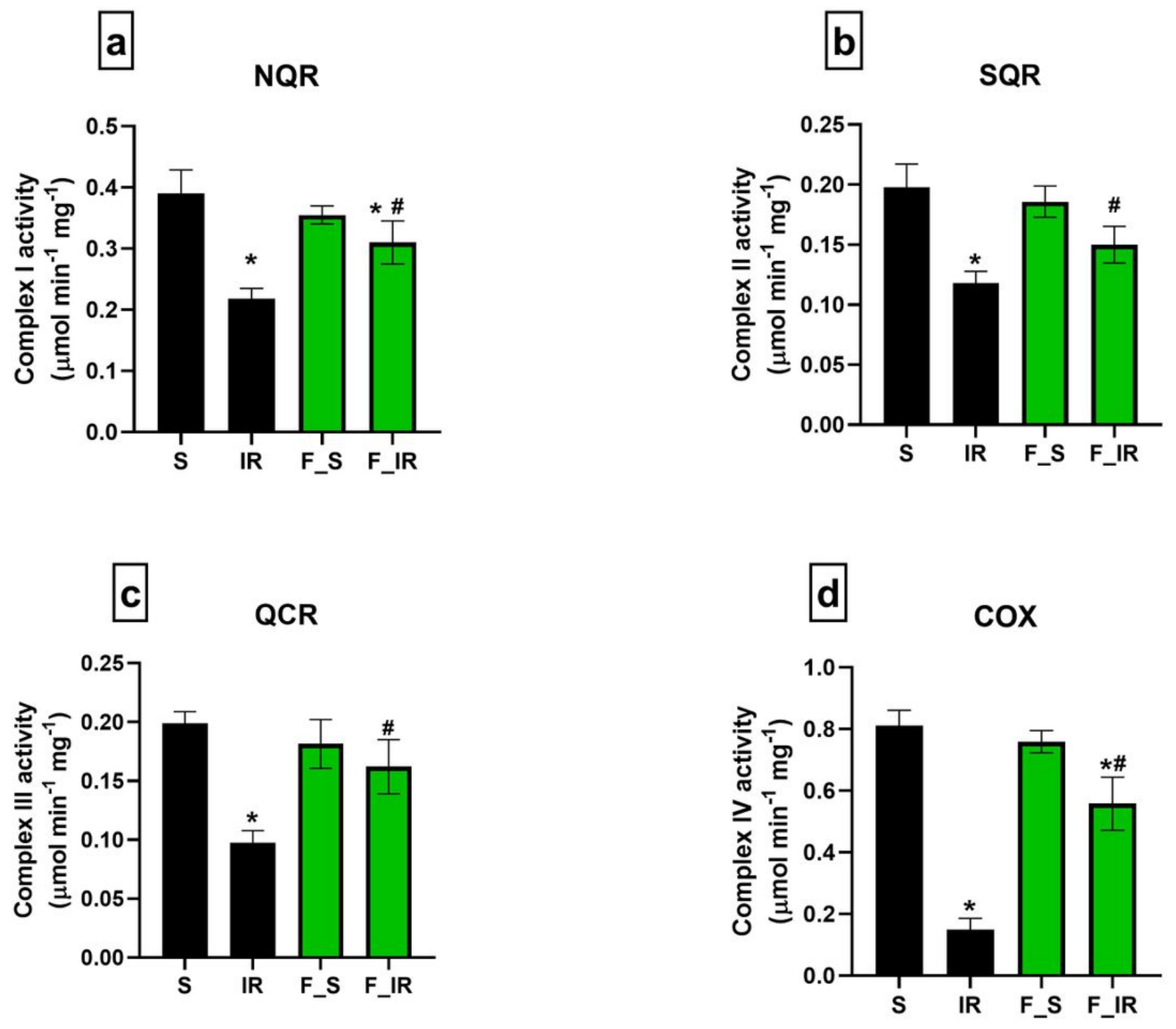

Figure 4

Mitochondrial ETC enzymes in sham and fisetin treated rats subjected to IR injury. (a) NQR activity, (b) SQR activity, (c) QCR activity and (d) COX activity. NQR activity was expressed as $\mu$ mol NADH oxidized min-1 mg -1 protein; SQR activity was expressed in $\mu$ mol DCPIP reduced min-1 mg -1 protein; QCR activity was expressed in $\mu \mathrm{mol}$ Cytochrome $\mathrm{C}$ reduced min-1 mg -1 protein; COX activity was expressed in $\mu \mathrm{mol}$ Cytochrome C oxidized min-1 mg -1 protein. ${ }^{*} p<0.05$ vs $S$, \# $p<0.05$ vs IR. The data are presented as mean \pm SD ( $n=6$ /group). S-Sham, IR-Ischemia-reperfusion, F_S- Fisetin sham, F_IR- Fisetin ischemiareperfusion. 

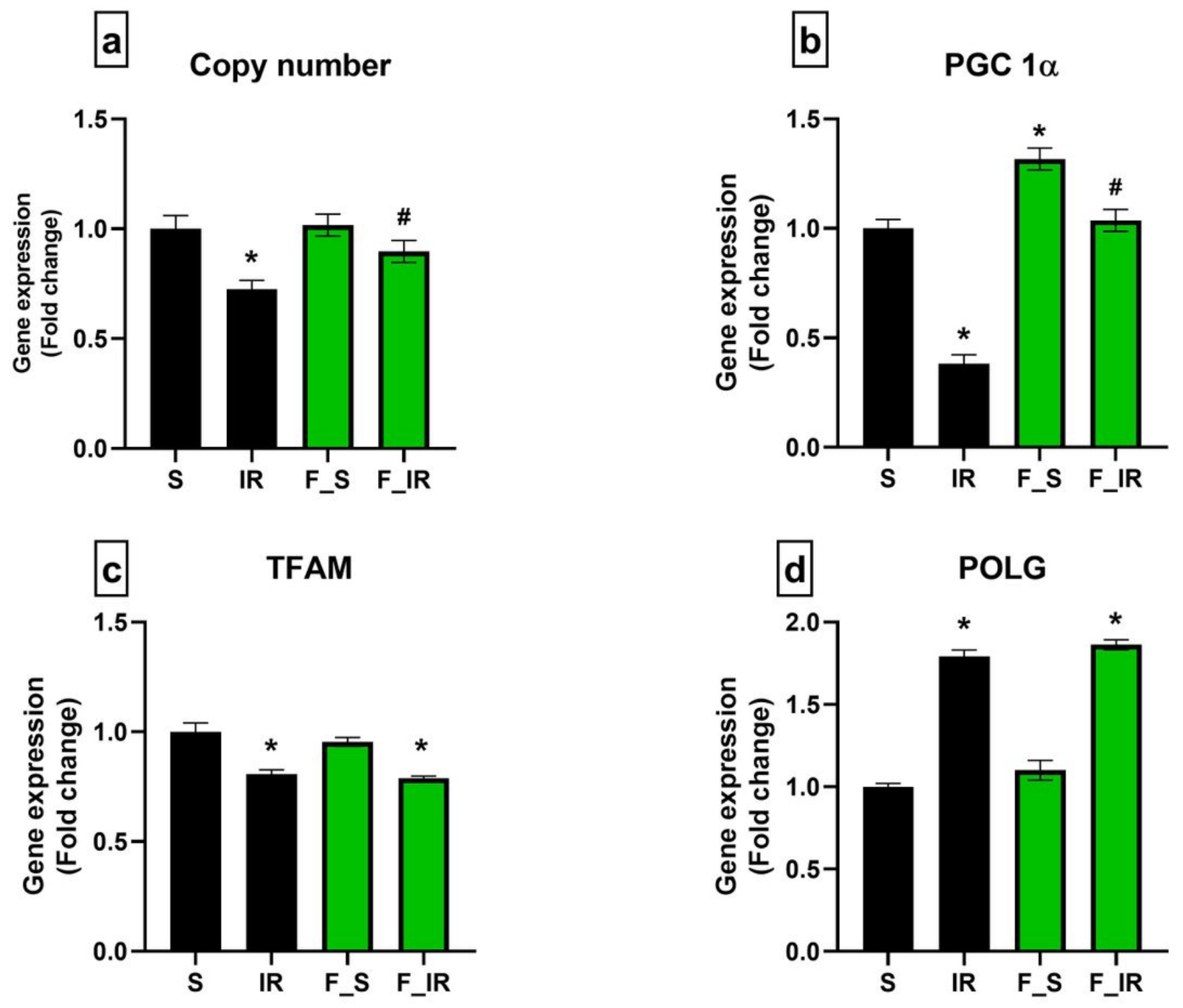

Figure 5

Gene expression analysis in sham and fisetin treated rats subjected to IR injury. Graph represents (a) mitochondrial copy number and (b) Pgc-1a expression (c) Tfam expression and (d) Polg in renal tissues. ${ }^{*} \mathrm{p}<0.05$ vs $\mathrm{S}, \# \mathrm{p}<0.05$ vs IR. The data are presented as mean $\pm S D(n=6 /$ group). S-Sham, IR-Ischemiareperfusion, F_S- Fisetin sham, F_IR- Fisetin ischemia-reperfusion. 

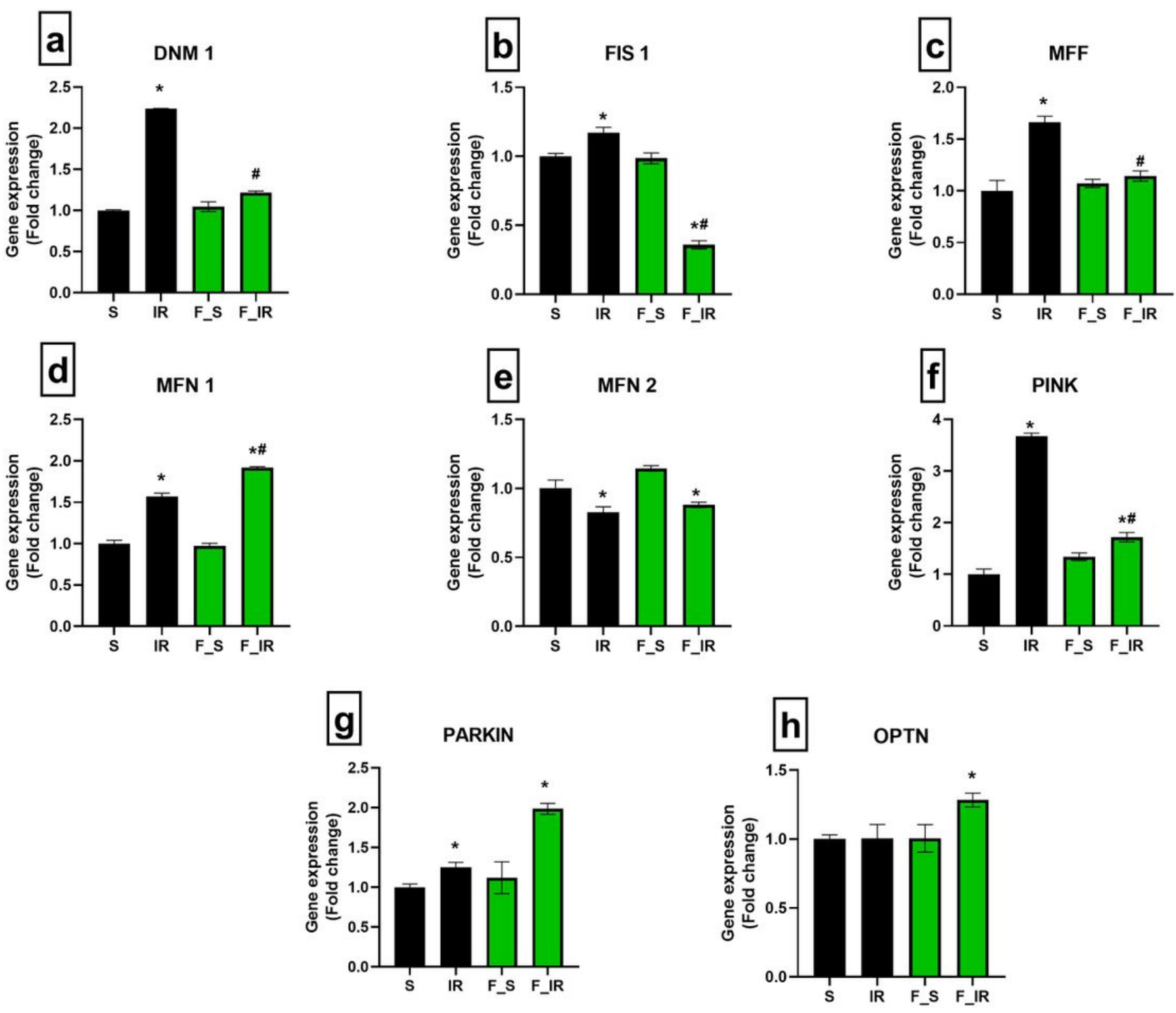

Figure 6

Expression analysis of genes encoding mitochondrial fission, fusion and mitophagy in renal tissues from sham and fisetin treated rats subjected to IR injury. The graph represents the mitochondrial fission genes (a) Dnm 1, (b) Fis 1, and (c) Mff; Mitochondrial fusion genes (d) Mfn 1 and (e) Mfn ; Mitophagic genes (f) Pink, (g) Parkin, and (h) Optn. ${ }^{*} p<0.05$ vs $S, \# p<0.05$ vs IR. The data are presented as mean \pm SD (n =6/group). S-Sham, IR-Ischemia-reperfusion, F_S- Fisetin sham, F_IR- Fisetin ischemia-reperfusion. 

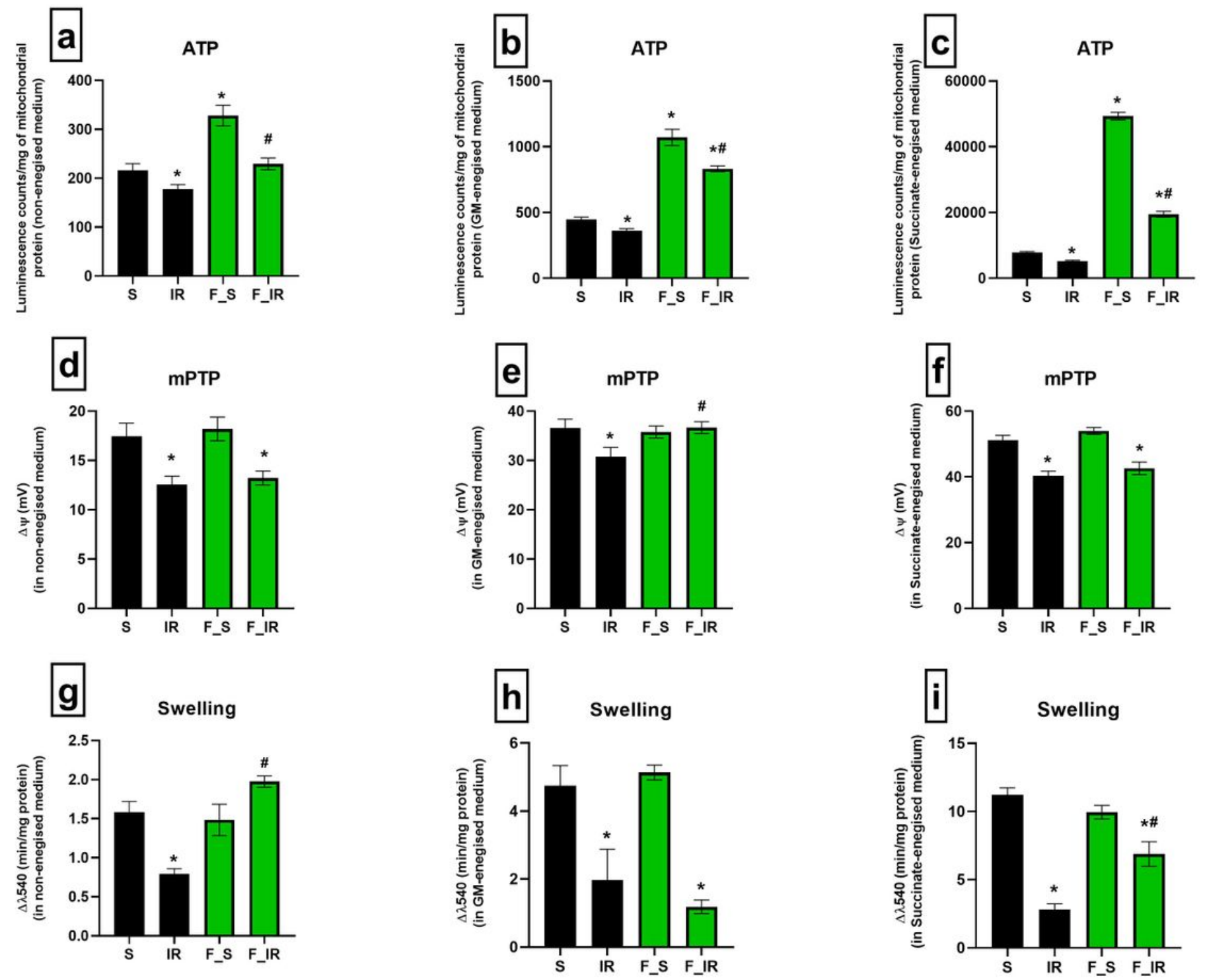

\section{Figure 7}

Analysis of changes in mitochondrial ATP production, membrane potential, and swelling in renal tissues from sham and fisetin treated rats subjected to IR injury. Graph represents measured ATP content using ATPlite luminescence kit, in (a) non-energized, (b) glutamate/malate energized and (c) succinate energized medium. (d), (e) and (f) represents mitochondrial membrane potential $(\Delta \square \mathrm{m})$ measured by Rhodamine 123 fluorescent dye in non-energized, succinate energized and glutamate/malamute energized medium respectively; $(\mathrm{g}),(\mathrm{h})$ and (i) represents calcium-induced mitochondrial swelling in nonenergized, succinate energized and glutamate/malamute energized medium respectively. ${ }^{*}<<0.05$ vs $S$, \# $p<0.05$ vs IR. The data are presented as mean \pm SD ( $n=6 /$ group). S-Sham, IR-Ischemia-reperfusion, F_SFisetin sham, F_IR- Fisetin ischemia-reperfusion. 

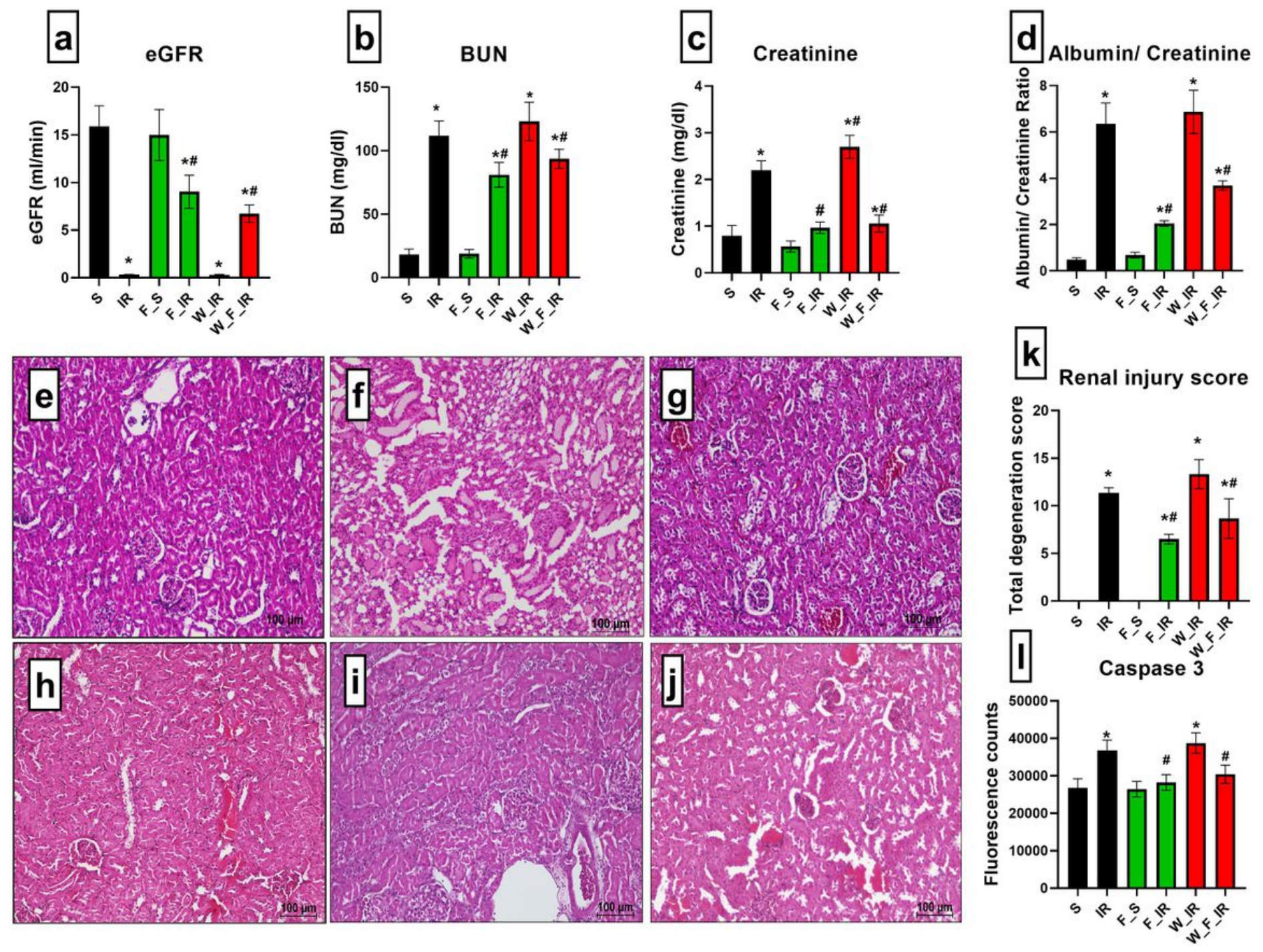

\section{Figure 8}

Renal physiology and injury analysis of wortmannin treated rats subjected to IR injury. Renal functional markers (a) eGFR, (b) Plasma creatinine, (c) Plasma BUN, and (d) Urinary albumin/Creatinine ratio. H\&E stained severe necrotic derangement images for (e) S, (f) IR, (g) F_S, (h) F_IR, (i) W_IR, and (j) W_F_IR and (k) Renal injury score from $\mathrm{H}$ and $\mathrm{E}$ staining. The images are presented at $10 \mathrm{x}$ magnification. Renal cell death analysis by (I) caspase activity. ${ }^{*} p<0.05$ vs $S, \# p<0.05$ vs IR. The data are presented as mean \pm SD ( $n=6 /$ group). S-Sham, IR-Ischemia-reperfusion, F_S- Fisetin sham, F_IR- Fisetin ischemia-reperfusion.

\section{Supplementary Files}

This is a list of supplementary files associated with this preprint. Click to download.

- FisetinFinalgraphpad.pzfx 